LA-UR-97-2880

Approted for public release; distribution is unlimited.

\title{
TITLE: A LOCAL DYNAMIC CORRELATION FUNCTION FROM INELASTIC NEUTRON SCATTERING
}

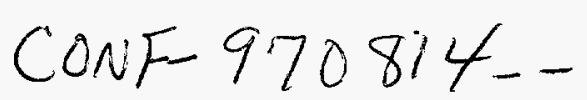

AUTHOR(S):

R.J. McQueeney, LANSCE 12

$\sum_{t=0}^{\infty} m^{\infty}$

1104 031997

$0 \%$
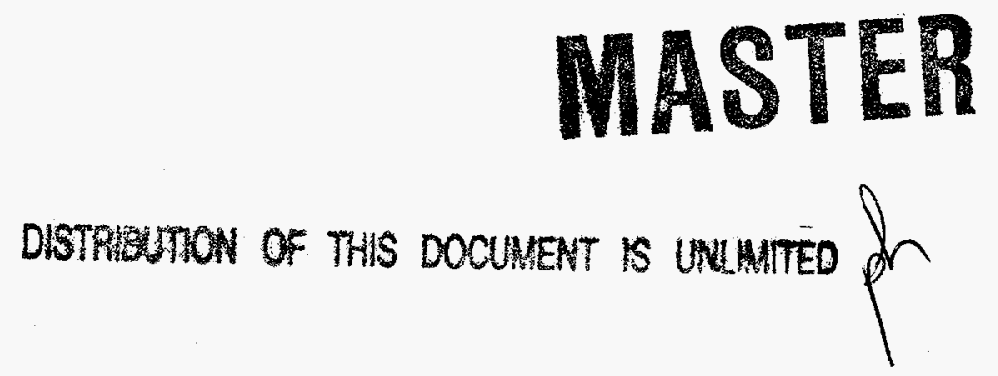

\section{Los Alamos}

NATIONAL LABORATORY

Los Alamos National Laboratory, an affirmative action/equal opportunity employer, is operated by the University of California for the U.S. Department of Energy under contract W-7405-ENG-36. By acceptance of this article, the publisher recognizes that the U.S. Government retains a nonexclusive, royalty-free license to publish or reproduce the published form of this contribution, or to allow others to do so, for U.S. Government purposes. The Los Alamos National Laboratory requests that the publisher identify this article as work performed under the auspices of the U.S. Department of Energy. The Los Alamos National Laboratory strongly supports academic freedom and a researcher's right to publish; as an institution, however, the Laboratory does not endorse the viewpoint of a publication or guarantee its technical correctness. 


\section{DISCLAIMER}

This report was prepared as an account of work sponsored by an agency of the United States Government. Neither the United States Government nor any agency thereof, nor any of their employees, makes any warranty, express or implied, or assumes any legal liability or responsibility for the accuracy, completeness, or usefulness of any information, apparatus, product, or process disclosed, or represents that its use would not infringe privately owned rights. Reference herein to any specific commercial product, process, or service by trade name, trademark, manufacturer, or otherwise does not necessarily constitute or imply its endorsement, recommendation, or favoring by the United States Government or any agency thereof. The views and opinions of authors expressed herein do not necessarily state or reflect those of the United States Government or any agency thereof. 


\section{DISCLAMIER}

Portions of this document msy be illegible in electronic image products. Images are produced from the best available original document. 
Version \#1: $7 / 13 / 97$

A Local Dynamic Correlation Function From Inelastic Neutron Scattering

\author{
R. J. McQueeney \\ MS H805, Los Alamos National Laboratory, Los Alamos, NM, 87545, USA
}

Abstract

Information about local and dynamic atomic correlations can be obtained from inelastic neutron scattering measurements by Fourier transform of the Q-dependent intensity oscillations at a particular frequency. A local dynamic structure function, $S(r, \omega)$, is defined from the dynamic scattering function, $S(Q, \omega)$, such that the elastic and frequencyintegrated limits correspond to the average and instantaneous pair-distribution functions, respectively. As an example, $\mathrm{S}(\mathrm{r}, \omega)$ is calculated for polycrystalline aluminum in a model where atomic motions are entirely due to harmonic phonons.

Keywords: inelastic neutron scattering, amorphous materials, local phenomena, phonons

$\begin{array}{ll}\text { Author: } & \text { Robert J. McQueeney } \\ \text { Address: } & \text { MS H805 } \\ & \text { Los Alamos National Laboratory } \\ & \text { Los Alamos, NM, 87545, USA } \\ \text { Fax: } & \text { (505)665-2676 } \\ \text { E-Mail: } & \text { mcqueeney@lanl.gov }\end{array}$


With the advent of high resolution, high intensity pulsed-neutron chopper spectrometers at spallation sources (such as PHAROS at LANSCE and MARI at ISIS), it has been possible to measure the dynamic scattering function, $\mathrm{S}(\mathrm{Q}, \omega)$, up to very large momentum transfers $\left(\mathrm{Q} \sim 20\right.$ or $\left.30 \AA^{-1}\right)$. The $\mathrm{Q}$ (momentum transfer)-dependence of the scattered intensity at constant frequency, $\omega$, gives information about the spatial distribution and relative displacements of atoms vibrating at that frequency. Due to the large range of $\mathrm{Q}$, one can ascertain the local dynamics contained in the Q-dependence by using Fourier methods to extract $S(r, \omega)$, a real-space dynamic structure function. This is defined by

$$
S(r, \omega)=\frac{1}{2 \pi^{2} r} \int_{0}^{\infty} Q d Q \sin (Q r)[S(Q, \omega)-B(Q, \omega)]
$$

where $\mathrm{B}(\mathrm{Q}, \omega)$ is the slowly varying background (due to incoherent, multiphonon and multiple scattering processes) which must be modeled (or fitted) and subtracted. Thus, $\mathrm{S}(\mathrm{Q}, \omega)-\mathrm{B}(\mathrm{Q}, \omega)$ is intended to represent the coherent inelastic scattering only.

This local, dynamic correlation function has two important limits. For elastic scattering, $S(r, \omega=0)$ corresponds to the average pair-distribution function (average PDF) and measures the probability of two atoms being separated by a distance $r$ when their positions are independently time-averaged. Integrating over all frequencies (including the elastic scattering) results in the instantaneous PDF, which measures the probability that two atoms are separated by $\mathrm{r}$ when their instantaneous positions are averaged over time. Any differences between the instantaneous and average PDFs imply the presence of local and dynamic atomic correlations. By obtaining the fully energy-resolved $S(r, \omega)$, one can determine the frequencies, atomic distances, and relative displacements of such correlations. In addition, one can quantify the strength of the correlations by comparison to the average PDF. 
The general concept of a real-space dynamic structure function was introduced by Carpenter and Pelizzari [1,2]. Experimentally, local dynamic functions similar to that defined by Eq.(1) have been obtained from inelastic neutron data by Arai, et al. and Hannon, et al. for amorphous Boron [3], amorphous ${\mathrm{g}-\mathrm{SiO}_{2}}_{2}$ [4-6], and a polycrystalline $\mathrm{YBa}_{2} \mathrm{Cu}_{3} \mathrm{O}_{7}$ superconductor $[6,7]$. However, the local dynamic function defined in the previous experiments includes a weighting factor of $\mathrm{Q}^{-2}$ in the Fourier transform. As Hannon et al. point out $[3,4]$, the result is not a true correlation function and the strength of the correlation cannot be estimated.

In order to examine these ideas, we calculate $S(r, \omega)$ for a simple polycrystalline material consisting of harmonic atomic displacements due to phonons. The elastic part of $\mathrm{S}(\mathrm{r}, \omega)$ is comprised of peaks corresponding to each coordination shell, which are broadened by the mean-squared atomic displacements, i.e. the Debye-Waller factor. Ignoring contributions from multiple, multiphonon, and incoherent scattering, the inelastic part of the real-space dynamic function $S(r, \omega)$ is shown below, due primarily to Carpenter et al.[1, 2].

$$
\begin{array}{r}
\mathrm{S}_{1}(\mathrm{r}, \omega)=\sum_{\ell \ell^{\prime}} \frac{\mathrm{b}_{\ell} \mathrm{b}_{\ell^{\prime}}}{\left\langle\mathrm{b}^{2}\right\rangle}\left\{-\mathrm{F}_{\ell \ell^{\prime}}^{\mathrm{B}}(\omega) \mathrm{K}_{2}\left(\mathrm{r}-\mathrm{R}_{\ell \ell^{\prime}}\right)\right. \\
\left.+\mathrm{F}_{\ell \ell^{\prime}}^{\mathrm{A}}(\omega)\left[\mathrm{K}_{0}\left(\mathrm{r}-\mathrm{R}_{\ell \ell^{\prime}}\right)+\mathrm{K}_{2}\left(\mathrm{r}-\mathrm{R}_{\ell \ell^{\prime}}\right)\right]\right\}
\end{array}
$$

where $\ell$ labels the atoms of mass $\mathrm{M}_{\ell}$ and (coherent) scattering length $\mathrm{b}_{\ell}$ and $\mathrm{R}_{\ell \ell^{\prime}}$ is the distance between two atoms.

$\mathrm{K}_{\mathrm{n}}$ are simple radial functions which only depend on the Debye-Waller factor. However, their peakshapes are not gaussian. When summing over frequency to produce the instantaneous PDF, $\mathrm{K}_{2}$ will 'narrow' or 'broaden' the average PDF, depending on the sign of $F^{B}$, and represents 'in-phase' and 'out-of-phase' atomic pair correlations, respectively. $K_{0}+K_{2}$ is 'displacive' and will shift a portion of the average PDF. $F^{A}$ is the 
displacement-displacement correlation function between two atoms, while $\mathrm{F}^{\mathrm{B}}$ measures the displacement correlations along the bond joining the two atoms. These functions are evaluated by averaging the correlations over constant frequency surfaces within the first Brillouin zone.

A simple model consisting of harmonic phonon vibrations aluminum is used illustrate these ideas. A Born-von Kármán model, including interactions up to the 8th nearest neighbor [8], were used to calculate the phonon frequencies and polarization vectors for approximately 67000 q-values within an octant of the FCC Brillouin zone.

The results of the calculation were used to determine the average and instantaneous PDFs at $\mathrm{T}=0 \mathrm{~K}$ for the first four coordination shells, as shown in Fig. 1. It is apparent that the local atomic correlations due to phonons sharpen the nearest neighbor peak significantly $(\sim 10 \%)$, while further neighbors show no significant correlated motion. Such a narrowing of the nearest neighbor instantaneous PDF peak implies a correlation which tends to move the atoms in-phase. It is interesting to note that this represents the contribution of the polycrystalline averaged thermal diffuse scattering to the instantaneous PDF.

This narrowing has been observed in neutron diffraction measurements of the instantaneous PDF in aluminum [9]. This is perhaps not very surprising. The thermal diffuse scattering is highly structured in Q-space, having power law singularities at Bragg points due to the large occupancy of low frequency phonons. Consequently, it will contribute additional structure to the PDF above that from the Bragg scattering.

The frequency dependence of $\mathrm{S}(\mathrm{r}=$ const., $\omega)$, shown for the first two coordination shells in Fig. 2, determines the frequency structure of correlated motions. In general, the peaks correspond to van Hove singularities in the phonon density-of-states and the sign is an indication of the in-phase or out-of-phase motion of an atomic pair. In this particular case, the dominant correlated motion arises from low frequency, long wavelength phonons which move nearby aluminum atoms in phase. At larger bond distances, $\mathrm{S}(\mathrm{r}=\mathrm{const} ., \omega)$ 
has more oscillations and the in-phase correlations are weaker since the phase difference between atoms is more sensitive to the phonon wavelength.

The author is grateful to R. A. Robinson and T. Egami for extremely helpful discussions.

This work was supported in part by the division of Basic Energy Sciences of the U.S. Department of Energy. 


\section{References}

[1] J. M. Carpenter and C. A. Pelizzari, Phys. Rev. B 12, 2391 (1975).

[2] J. M. Carpenter and C. A. Pelizzari, Phys. Rev. B 12, 2397 (1975).

[3] A. C. Hannon, et al., Nucl. Instr. Meth. A 354, 96 (1995).

[4] A. C. Hannon, et al., J. Non-cryst. Solids 150, 239 (1992).

[5] M. Arai, et al., Physica B 180\&181, 779 (1992).

[6] M. Arai, et al., J. Non-cryst. Solids 192\&193, 230 (1995).

[7] M. Arai, et al., J. Supercond. 7, 415 (1994).

[8] G. Gilat and R. M. Nicklow, Phys. Rev. 143, 489 (1966).

[9] T. Egami, private communication 
Figure Captions

Fig. 1 - The calculated average, instantaneous, and inelastic PDFs for the first four nearest neighbors in aluminum.

Fig. 2 - The local dynamic correlation function $S(r=c o n s t, \omega)$ calculated for the first two pair distances in aluminum. 


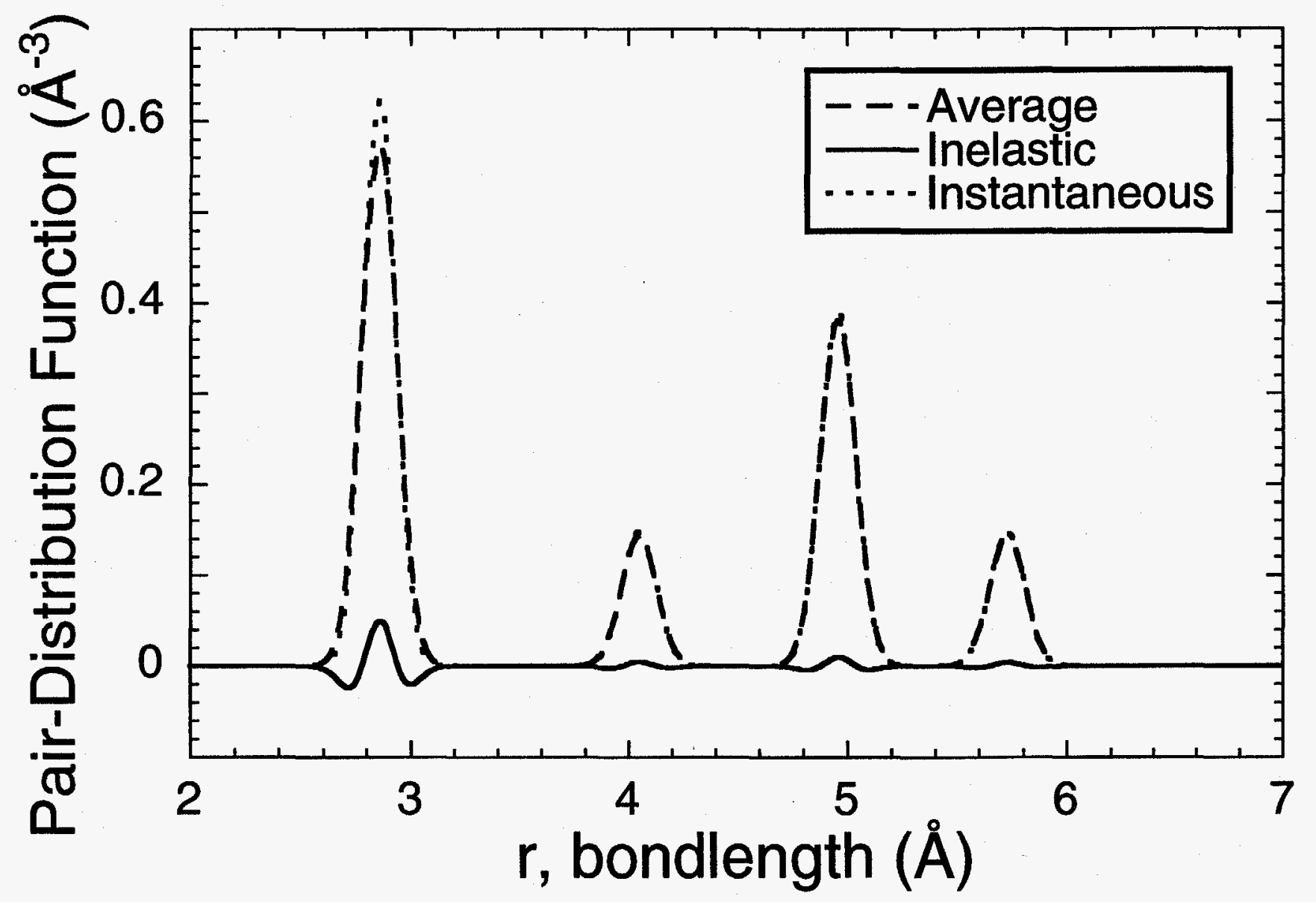




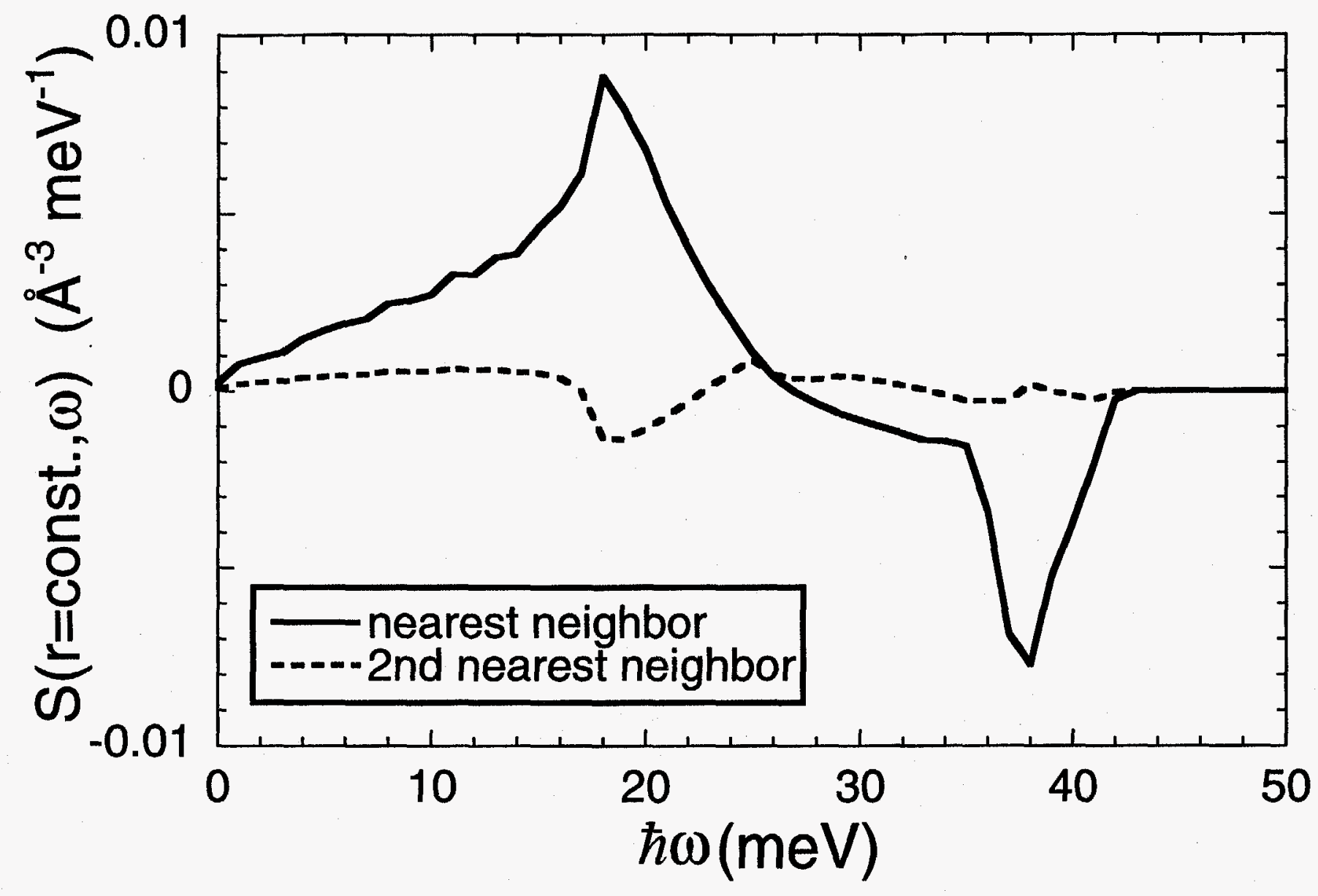

